\title{
Multisymplectic method for the Camassa-Holm equation
}

\author{
Yu Zhang, Zi-Chen Deng* and Wei-Peng Hu
}

"Correspondence:

dweifan@nwpu.edu.cn

School of Mechanics, Civil Engineering and Architecture,

Northwestern Polytechnical University, Xi'an, Shannxi 710072, P.R. China

\section{Springer}

\begin{abstract}
The Camassa-Holm equation, a completely integrable evolution equation, contains rich geometric structures. For the existence of the bi-Hamiltonian structure and the so-called peaked wave solutions, considerable interest has been aroused in the last several decades. Focusing on local geometric properties of the peaked wave solutions for the Camassa-Holm equation, we propose the multisymplectic method to simulate the propagation of the peaked wave in this paper. Based on the multisymplectic theory, we present a multisymplectic formulation of the Camassa-Holm equation and the multisymplectic conservation law. Then, we apply the Euler box scheme to construct the structure-preserving scheme of the multisymplectic form. Numerical results show the merits of the multisymplectic scheme constructed, especially the local conservative properties on the wave form in the propagation process.
\end{abstract}

MSC: 35Q51; 37K10; 65P10

Keywords: multisymplectic method; Camassa-Holm equation; conservation law; peaked wave solution

\section{Introduction}

The $b$-family equation [1] with the uniform form

$$
u_{t}-u_{x x t}+(b+1) u u_{x}=b u_{x} u_{x x}+u u_{x x x}
$$

as a kind of shallow water equations including famous Camassa-Holm equation $(b=2)$ and Degasperis-Procesi equation $(b=3)$, has received considerable interest during the last decade.

This partial differential equation (PDE) is a one-dimensional version of the active fluid transport that has been proved to be integrable and is called an infinite bi-Hamiltonian system. In the early 1990s, the Camassa-Holm equation was proposed by Camassa and Holm [2] and possesses an infinite number of conservation laws in involution for the existence of the bi-Hamiltonian. In their work, the discontinuity in the first derivative at its peak of the soliton solution has been given as a limiting form. Constantin [3] proved that the Camassa-Holm equation is completely integrable. Fisher and Schiff [4] showed that the Camassa-Holm equation has an infinite number of local conserved quantities by a Miura-Gardner-Kruskal-type construction. In the late 1990s, based on the method of asymptotic integrability, Degasperis and Procesi derived the Degasperis-Procesi equation.

(c) 2016 Zhang et al. This article is distributed under the terms of the Creative Commons Attribution 4.0 International License (http://creativecommons.org/licenses/by/4.0/), which permits unrestricted use, distribution, and reproduction in any medium, provided you give appropriate credit to the original author(s) and the source, provide a link to the Creative Commons license, and indicate if changes were made. 
Since then, there are many works reported on this equation: Degasperis et al. [5] proved that the Degasperis-Procesi equation is also integrable and admits multipeakon solutions. Lundmark [6] proposed an inverse scattering approach for seeking $n$-peakon solutions and presented the jump discontinuity of the shockpeakon solution for the DegasperisProcesi equation firstly. Coclite et al. [7] constructed several numerical schemes for capturing discontinuous solutions of the Degasperis-Procesi equation and proved that the schemes converge to entropy solutions. Later, Coclite et al. [8] studied the process of singularity formation for the $b$-family equation and presented the numerical results of the Degasperis-Procesi equation.

The aforementioned works for the $b$-family equation on the peakon solutions are conducted only in two special cases ( $b=2$ and $b=3$ ) because the equation is integrable only in these two cases, which means that the Camassa-Holm and the Degasperis-Procesi equations may contain many interesting geometric properties. The aim of this paper is to use the multisymplectic algorithms for the numerical simulation on the peakon solution of the Camassa-Holm equation [2]. When $b=2$, the Camassa-Holm equation reads

$$
u_{t}-u_{x x t}+3 u u_{x}=2 u_{x} u_{x x}+u u_{x x x} .
$$

For Eq. (2), there are some special solitons called peakon solutions, which are known to be rich in geometric structures and to have nonsmooth traveling wave solutions.

Many numerical methods have been used to solve the Camassa-Holm equation. For example, Coclite et al. $[9,10]$ derived a finite difference scheme for the Camassa-Holm equation that can handle general $H^{1}$ initial data. Xu and Shu [11] developed a local discontinuous Galerkin method that enhances some nonlinear high-order derivatives to solve the Camassa-Holm equation. Matsuo [12] presented a new $G_{2}$-conserving Galerkin scheme for the Camassa-Holm equation. Kalisch and Lenells [13] used a pseudospectral scheme to investigate the traveling-wave solutions of the Camassa-Holm equation. Subsequently, Kalisch and Raynaud [14] investigated the convergence of the pseudospectral scheme of the Camassa-Holm equation. However, reports on the numerical analysis of the CamassaHolm equation based on structure-preserving method are limited: Cohen et al. [15] presented two multisymplectic formulations for the Camassa-Holm equation to simulate the peakon and peakon-antipeakon collisions. Zhu et al. [16] developed a multisymplectic wavelet collocation method for solving multisymplectic Hamiltonian system with periodic boundary conditions and showed high accuracy and good conservation properties of the numerical method.

As a kind of structure-preserving methods, the multisymplectic integrator can preserve the intrinsic properties of the infinite-Hamiltonian systems [17-19] in each time step. It is well known that the peakon is a wave with local geometric property of the Camassa-Holm equation that can be investigated by the multisymplectic integrator because the multisymplectic method pays more attention to the local geometric properties of the systems.

The rest of this paper is organized as follows. In Section 2, we deduce the multisymplectic formulation of the Camassa-Holm equation. In Section 3, we construct the multisymplectic Euler box scheme to simulate the peaked wave of the Camassa-Holm equation. In Section 4, the numerical results are carried out to show that this scheme is of high accuracy and has good invariant-conserving properties. Finally, some conclusions are given in the last section. 


\section{The multisymplectic formulations of the Camassa-Holm equation}

The multisymplectic integrator algorithm has aroused considerable interest since it was presented in 1997 [17]. As a very robust framework, this algorithm has many advantages in the process of dealing with some conservative PDE systems, such as high accuracy and good long-time numerical behavior. The basic idea of a multisymplectic integrator is that the numerical scheme is designed to preserve the multisymplectic form at each time step [17]. Because the multisymplectic idea presented a new requirement for a numerical algorithm, it has aroused an important revolution in numerical methods for PDEs. Based on the multisymplectic idea, multisymplectic methods have been applied to approximate some famous conservative PDEs, which are exactly in existence of multisymplectic forms, such as nonlinear wave equations [18, 20, 21], Schrödinger equations [22-25], KdV equations [26-28], Boussinesq equations [29, 30], Maxwell equations [31-33], $b$-family equations [15, 16, 34], Kawahara-type equation [35], etc. In this section, we give the multisymplectic form of the Camassa-Holm equation.

After the complete Legendre transform, a wide range of PDEs can be written in the multisymplectic canonical form

$$
\mathbf{M} \mathbf{z}_{t}+\mathbf{K} \mathbf{z}_{x}=\nabla_{z} S(\mathbf{z}), \quad \mathbf{z} \in \mathbf{R}^{d}
$$

where $\mathbf{M}$ and $\mathbf{K} \in \mathbf{R}^{d \times d}$ are skew-symmetric matrices, and $S: \mathbf{R}^{d} \rightarrow R$ is a smooth function of the state variable $\mathbf{z}$. The above system has several conservation laws, which have been proposed in [17]. According to multisymplectic theory [17, 19], the multisymplectic conservation law can be expressed as

$$
\partial_{t} \omega+\partial_{x} \kappa=0,
$$

where the presymplectic forms $\omega$ and $\kappa$ are

$$
\omega=\frac{1}{2} \mathrm{~d} \mathbf{z} \Lambda \mathbf{M d z}, \quad \kappa=\frac{1}{2} \mathrm{~d} \mathbf{z} \Lambda \mathbf{K} \mathrm{d} \mathbf{z},
$$

where $\Lambda$ is the wedge product operator.

With the skewsymmetric matrices $\mathbf{M}$ and $\mathbf{K}$, we can also derive the local conservation laws of energy and momentum by taking the inner product of multisymplectic form (3) with $\partial_{t} \mathbf{z}$ and $\partial_{x} \mathbf{z}$, respectively. The local energy conservation law is

$$
\partial_{t} e+\partial_{x} f=0
$$

where the density functions are

$$
e=S(\mathbf{z})-\frac{1}{2} \mathbf{z}^{\mathrm{T}} \mathbf{K} \partial_{x} \mathbf{z}, \quad f=\frac{1}{2} \mathbf{z}^{\mathrm{T}} \mathbf{K} \partial_{t} \mathbf{z},
$$

and the local momentum conservation law is

$$
\partial_{t} h+\partial_{x} g=0
$$


where the density functions are

$$
h=\frac{1}{2} \mathbf{z}^{\mathrm{T}} \mathbf{M} \partial_{x} \mathbf{z}, \quad g=S(\mathbf{z})-\frac{1}{2} \mathbf{z}^{\mathrm{T}} \mathbf{M} \partial_{t} \mathbf{z} .
$$

By introducing the following canonical momenta and setting the state variable $\mathbf{z}=$ $[u, \varphi, w, \psi, v]^{\mathrm{T}} \in R^{5}$ we have

$$
\begin{aligned}
& u_{t}=-w_{x}, \quad \varphi_{x}=u, \\
& u_{x}=v, \quad \frac{1}{2} u_{t}=-u v+\psi .
\end{aligned}
$$

The Camassa-Holm equation (2) can be reformulated as a multisymplectic canonical form (3) with the skew-symmetric matrices

$$
\mathbf{M}=\left[\begin{array}{ccccc}
0 & \frac{1}{2} & 0 & 0 & -\frac{1}{2} \\
-\frac{1}{2} & 0 & 0 & 0 & 0 \\
0 & 0 & 0 & 0 & 0 \\
0 & 0 & 0 & 0 & 0 \\
\frac{1}{2} & 0 & 0 & 0 & 0
\end{array}\right], \quad \mathbf{K}=\left[\begin{array}{ccccc}
0 & 0 & 0 & -1 & 0 \\
0 & 0 & -\frac{1}{2} & 0 & 0 \\
0 & \frac{1}{2} & 0 & 0 & 0 \\
1 & 0 & 0 & 0 & 0 \\
0 & 0 & 0 & 0 & 0
\end{array}\right]
$$

and the Hamiltonian function $S(\mathbf{z})=\frac{1}{2} u w-\frac{1}{2} u^{3}-\frac{1}{2} u v^{2}+v \psi$.

According to Eq. (4), a multisymplectic conservation law for the Camassa-Holm equation can be obtained:

$$
\frac{\partial}{\partial t}[\mathrm{~d} u \Lambda \mathrm{d} \varphi+\mathrm{d} v \Lambda \mathrm{d} u]+\frac{\partial}{\partial x}(2 \mathrm{~d} \psi \Lambda \mathrm{d} u+\mathrm{d} w \Lambda \mathrm{d} \varphi)=0
$$

\section{Multi-symplectic Euler box scheme for the Camassa-Holm equation}

A numerical scheme is multisymplectic if and only if the numerical scheme preserves the discrete multisymplectic conservation law [19]. As a widely used numerical scheme, the Euler box scheme [36] for the PDEs (3) has been proved to be multisymplectic. An integrator form satisfying a discrete multisymplectic conservation law can be obtained by introducing the splitting of the two matrices $\mathbf{M}$ and $\mathbf{K}$ defined as $\mathbf{M}=\mathbf{M}_{+}+\mathbf{M}_{-}$and $\mathbf{K}=\mathbf{K}_{+}+\mathbf{K}_{-}$, where $\mathbf{M}_{+}^{\mathrm{T}}=-\mathbf{M}_{-}$and $\mathbf{K}_{+}^{\mathrm{T}}=-\mathbf{K}_{-}$.

Let $t_{i}, i=1,2, \ldots ; x_{n}, n=1,2, \ldots$ be the grids of the integral domain, $\Delta t=t_{i+1}-t_{i}$ be the time step length, and $\Delta x=x_{n+1}-x_{n}$ be the spatial step length. Then the corresponding Euler box discrete scheme of Eq. (3) reads

$$
\mathbf{M}_{+} \delta_{t}^{+} \mathbf{z}^{n, i}+\mathbf{M}_{-} \delta_{t}^{-} \mathbf{z}^{n, i}+\mathbf{K}_{+} \delta_{x}^{+} \mathbf{z}^{n, i}+\mathbf{K}_{-} \delta_{x}^{-} \mathbf{z}^{n, i}=\nabla_{z} S\left(\mathbf{z}^{n, i}\right)
$$

where

$$
\begin{aligned}
\delta_{t}^{+} \mathbf{z}^{n, i}=\frac{\mathbf{z}^{n, i+1}-\mathbf{z}^{n, i}}{\Delta t}, & \delta_{x}^{+} \mathbf{z}^{n, i}=\frac{\mathbf{z}^{n+1, i}-\mathbf{z}^{n, i}}{\Delta x}, \\
\delta_{t}^{-} \mathbf{z}^{n, i}=\frac{\mathbf{z}^{n, i}-\mathbf{z}^{n, i-1}}{\Delta t}, & \delta_{x}^{-} \mathbf{z}^{n, i}=\frac{\mathbf{z}^{n, i}-\mathbf{z}^{n-1, i}}{\Delta x} .
\end{aligned}
$$


Now, we will construct the multisymplectic Euler box scheme for the Camassa-Holm equation in detail. Taking $\mathbf{M}_{+}=\frac{1}{2} \mathbf{M}$ and $\mathbf{K}_{+}=\frac{1}{2} \mathbf{K}$, the splitting of $\mathbf{M}$ and $\mathbf{K}$ reads

$$
\mathbf{M}_{+}=\left[\begin{array}{ccccc}
0 & \frac{1}{4} & 0 & 0 & -\frac{1}{4} \\
-\frac{1}{4} & 0 & 0 & 0 & 0 \\
0 & 0 & 0 & 0 & 0 \\
0 & 0 & 0 & 0 & 0 \\
\frac{1}{4} & 0 & 0 & 0 & 0
\end{array}\right], \quad \mathbf{K}_{+}=\left[\begin{array}{ccccc}
0 & 0 & 0 & -\frac{1}{2} & 0 \\
0 & 0 & -\frac{1}{4} & 0 & 0 \\
0 & \frac{1}{4} & 0 & 0 & 0 \\
\frac{1}{2} & 0 & 0 & 0 & 0 \\
0 & 0 & 0 & 0 & 0
\end{array}\right] .
$$

Thus, the expanded form of the Euler box scheme for the multisymplectic structure (3) with the particular $\mathbf{M}_{+}$and $\mathbf{K}_{+}$is

$$
\begin{aligned}
& \frac{1}{4}\left(\delta_{t}^{+} \varphi^{n, i}+\delta_{t}^{-} \varphi^{n, i}\right)-\frac{1}{4}\left(\delta_{t}^{+} v^{n, i}+\delta_{t}^{-} v^{n, i}\right)-\frac{1}{2}\left(\delta_{x}^{+} \psi^{n, i}+\delta_{x}^{-} \psi^{n, i}\right) \\
& =\frac{1}{2} w^{n, i}-\frac{3}{2}\left(u^{n, i}\right)^{2}-\frac{1}{2}\left(v^{n, i}\right)^{2} \\
& -\frac{1}{4}\left(\delta_{t}^{+} u^{n, i}+\delta_{t}^{-} u^{n, i}\right)-\frac{1}{4}\left(\delta_{x}^{+} w^{n, i}+\delta_{x}^{-} w^{n, i}\right)=0 \\
& \frac{1}{4}\left(\delta_{x}^{+} \varphi^{n, i}+\delta_{x}^{-} \varphi^{n, i}\right)=\frac{1}{2} u^{n, i} \\
& \frac{1}{2}\left(\delta_{x}^{+} u^{n, i}+\delta_{x}^{-} u^{n, i}\right)=v^{n, i} \\
& \frac{1}{4}\left(\delta_{t}^{+} u^{n, i}+\delta_{t}^{-} u^{n, i}\right)=-u^{n, i} v^{n, i}+\psi^{n, i} .
\end{aligned}
$$

It involves considerable effort in computing the auxiliary variables $\varphi, w, \psi, v$ and difficulty in defining their initial values. So, all the additional variables $\varphi, w, \psi, v$ are eliminated by straightforward substitution and algebraic manipulations. Then the following multisymplectic Euler box scheme only in terms of the variable $u$ is obtained:

$$
\delta_{t} u^{n, i}-\delta_{x}^{2} \delta_{t} u^{n, i}-\delta_{x}^{2}\left(u^{n, i} \delta_{x} u^{n, i}\right)+\frac{3}{2} \delta_{x}\left(u^{n, i}\right)^{2}+\frac{1}{2} \delta_{x}\left(\delta_{x} u^{n, i}\right)^{2}=0,
$$

where the centered divided differences $\delta_{t}, \delta_{x}$ are

$$
\delta_{t}=\frac{1}{2}\left(\delta_{t}^{+}+\delta_{t}^{-}\right), \quad \delta_{x}=\frac{1}{2}\left(\delta_{x}^{+}+\delta_{x}^{-}\right) .
$$

Expanding Eq. (10), we can obtain a thirteen-point multisymplectic scheme.

\section{Numerical results on the peaked wave solution of the Camassa-Holm equation}

A soliton is a solitary wave caused by a delicate balance between the nonlinear and dispersive effects in the medium. It maintains its shape while travels at a constant speed theoretically. However, in the absence of the dispersion term, the parameter $b$ in the Camassa-Holm equation introduces the nonlinear and nonlocal balance. The nonlinear and nonlocal balance can still produce a soliton solution, which called the peakon solution, moving in the medium as

$$
u(t, x)=c e^{-|x-c t|}, \quad c>0
$$


where $c$ is the wave speed. Obviously, the derivative of Eq. (11) has a jump discontinuity at its peak.

In this section, we reproduce the peaked wave solution of the Camassa-Holm equation and investigate the global conserved quantities for the peakon solution of the CamassaHolm equation. In the following numerical experiments, the scheme (10) is used to simulate the peakon solution of the Camassa-Holm equation given by

$$
u=e^{-|x-t|}
$$

The derivative of Eq. (12) at $t=0$ can be obtained directly:

$$
u_{x}=-\operatorname{sgn}(x) e^{-|x|}
$$

with the limits

$$
\lim _{x \rightarrow 0^{+}} u_{x}=-1, \quad \lim _{x \rightarrow 0^{-}} u_{x}=1,
$$

which describe the jump discontinuity at the peakon position.

\subsection{Numerical results on peaked wave solution}

We take the time step length $\Delta t=0.005$ and space step length $\Delta x=0.005$ in the domain $D:(t, x) \in[0,2] \times[-5,5]$. Consider the following initial condition:

$$
u(0, x)=e^{-|x|} .
$$

As is well known, the peakon solution given by Eq. (12) for the Camassa-Holm equation is a sharp solitary wave with the unit amplitude traveling from left to right. Using the Euler box scheme (10), we obtained the waveforms of the peakon solution at moments $t=0,0.5,1$, and 1.5 shown in Figure 1.

From Figure 1 we can conclude that the wave shape of the numerical solution is smooth enough and does not change in the process of propagation. Thus, the multisymplectic Euler box scheme for the Camassa-Holm equation can be successfully used to simulate the peakon solution.

To investigate the geometric properties of the scheme (10), Figure 2 gives the errors between the numerical solution and exact solution at the moments $t=0.5,1$, and 1.5 , and the numerical and exact results on some grid points at the moment $t=1$ are further shown in Table 1.

Comparing the numerical result with the exact solution, we can conclude that the multisymplectic Euler box scheme (10) can simulate the peaked traveling wave with small numerical errors. As shown in Figure 2, the maximum magnitude of the errors is up to $10^{-3}$, which only appear at the points near the peakon, whereas on the grid points far away from the peakon, the magnitude of the errors are less than $10^{-6}$, as shown in Table 1.

Some numerical values of the left and right limits of $u_{x}$ at the peakon position at different times are presented in Table 2.

From Table 2 we find that the value of $\lim _{x \rightarrow 0^{+}} u_{x}$ is approximately 1 and $\lim _{x \rightarrow 0^{-}} u_{x}$ is about -1 in the process of the peaked wave propagation. Similarly to the theoretical results 

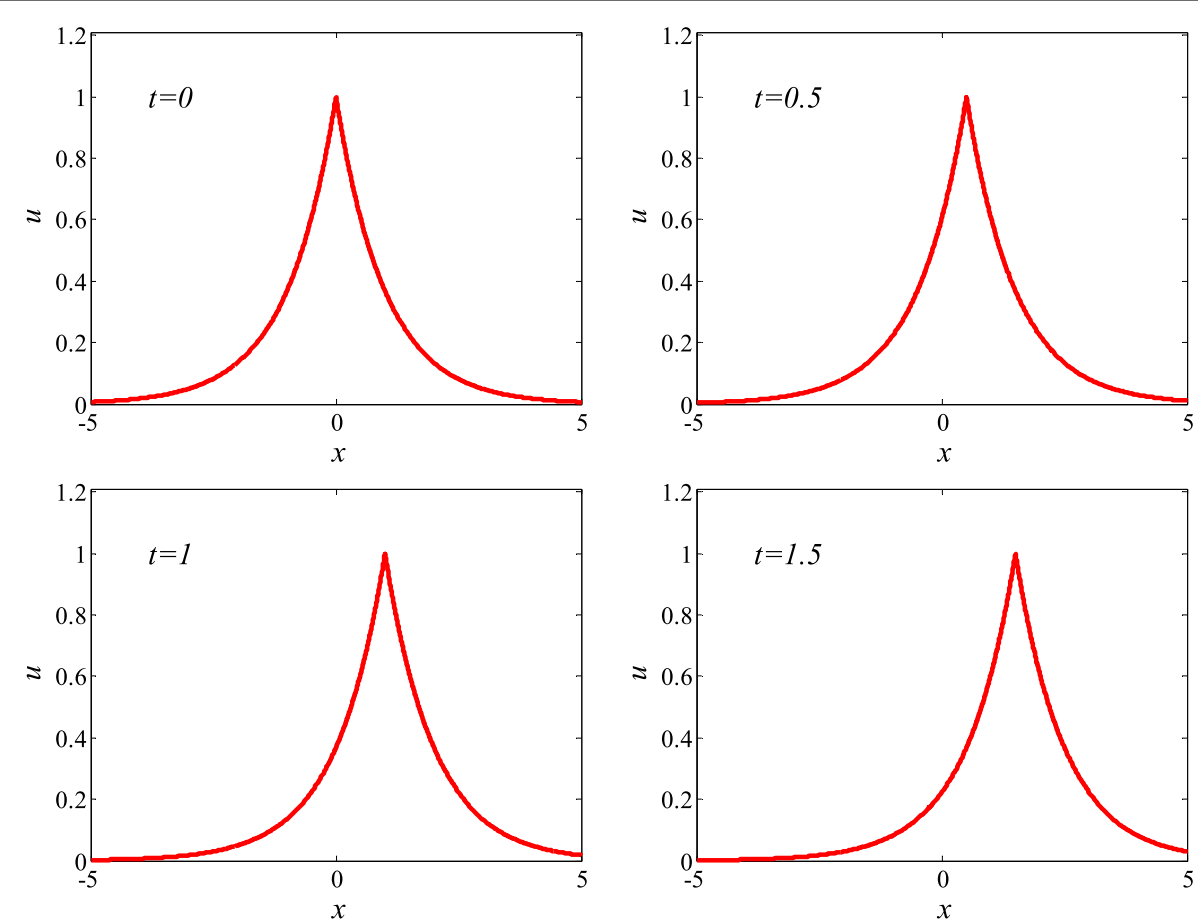

Figure 1 Waveforms of the peakon solution.

Figure 2 The errors between the numerical solution and peakon solution.

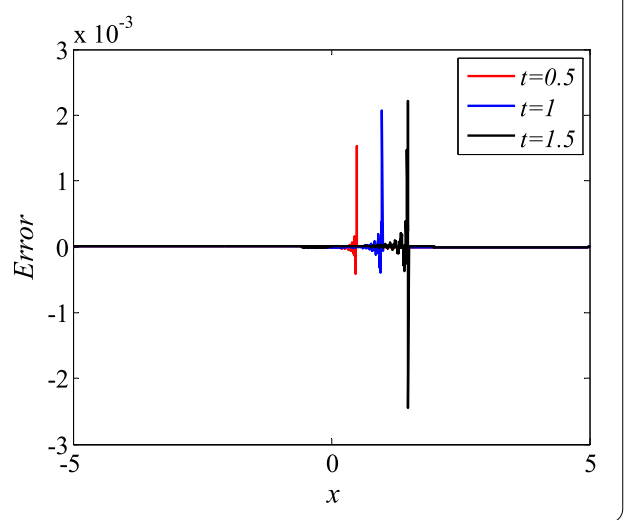

Table 1 The numerical and exact data on some grid points at $t=1$

\begin{tabular}{|c|c|c|c|c|c|}
\hline$x$ & Numerical data & Exact data & $x$ & Numerical data & Exac \\
\hline-4.5 & 0.0040867762 & 0.0040867714 & 0.5 & 0.6065338144 & 0.606 \\
\hline-4 & 0.0067379718 & 0.0067379470 & 1 & 1.0000083119 & 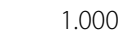 \\
\hline-3.5 & 0.0111090695 & 0.0111089965 & 1.5 & 0.6065303657 & \\
\hline-3 & 0.0183158110 & 0.0183156389 & 2 & 0.3678784135 & \\
\hline-2.5 & 0.0301977431 & 0.0301973834 & 2.5 & 0.2231294390 & \\
\hline-2 & 0.0497877582 & 0.0497870684 & 3 & 0.1353348944 & \\
\hline-1.5 & 0.0820862237 & 0.0820849986 & 3.5 & 0.0820848236 & \\
\hline-1 & 0.1353372802 & 0.1353352832 & 4 & 0.0497870068 & \\
\hline-0.5 & 0.2231319769 & 0.2231301601 & 4.5 & 0.0301973713 & \\
\hline \multicolumn{6}{|c|}{ Numerical values of $u_{x}$ at the peakon position } \\
\hline & \multicolumn{2}{|c|}{$t=0.2$} & $t=1.0$ & $t=1.4$ & $t=1.8$ \\
\hline & $\begin{array}{l}\lim _{x \rightarrow 0^{+}} u_{x} \\
\lim _{x \rightarrow 0^{-}} u_{x}\end{array}$ & $\begin{array}{r}0.97305 \\
-0.99753\end{array}$ & $\begin{array}{r}1.01050 \\
-0.99755\end{array}$ & $\begin{array}{r}0.98411 \\
-0.99759\end{array}$ & $\begin{array}{r}0.97879 \\
-0.99765\end{array}$ \\
\hline
\end{tabular}


shown in Eq. (14), the discontinuity phenomenon on the peakon of the soliton solution is reproduced numerically.

As an explicit scheme, such a high precision of the multisymplectic Euler box scheme is worth being further verified in the future. The results illustrate the structure-preserving properties of the multisymplectic integration.

\subsection{Three global conserved quantities for the Camassa-Holm equation}

Referring to the bi-Hamiltonian structure of the Camassa-Holm equation [2], the Camassa-Holm equation has an infinite sequence of conservation laws that insure the existence of the peaked wave during the propagation process. The first three important global conserved quantities associated with the Camassa-Holm equation are $[2,15,16]$

$$
\left\{\begin{array}{l}
H_{0}=\int u \mathrm{~d} x, \\
H_{1}=\frac{1}{2} \int\left(u^{2}+u_{x}^{2}\right) \mathrm{d} x, \\
H_{2}=\frac{1}{2} \int\left(u^{3}+u u_{x}^{2}\right) \mathrm{d} x .
\end{array}\right.
$$

As shown in Eq. (16), $H_{0}$ and $H_{1}$ represent the conservation laws for the mass and momentum with the process of the propagation, respectively. The third conserved quantity $\mathrm{H}_{2}$ is obtained by an action principle, which expressed in terms of a velocity potential [2]. The conservation laws for the Degasperis-Procesi equation are much weaker than the conservation laws for the Camassa-Holm equation. Thus, the orbital stability of DegasperisProcesi peaked solitons is more subtle [37].

Note here that the local conservation laws of energy and momentum for the CamassaHolm equation according to Eqs. (5) and (6) are, respectively,

$$
\begin{aligned}
& \partial_{t}\left[S(\mathbf{z})-\frac{1}{2}\left(\psi u_{x}+\frac{1}{2} w \varphi_{x}-\frac{1}{2} \varphi w_{x}-u \psi_{x}\right)\right] \\
& \quad+\partial_{x}\left[\frac{1}{2}\left(\psi u_{t}+\frac{1}{2} w \varphi_{t}-\frac{1}{2} \varphi w_{t}-u \psi_{t}\right)\right]=0, \\
& \partial_{t}\left[\frac{1}{4}\left(-u_{x} \varphi+u_{x} v+u \varphi_{x}-u v_{x}\right)\right]+\partial_{x}\left[S(\mathbf{z})+\frac{1}{4}\left(\varphi u_{t}-v u_{t}-u \varphi_{t}+u v_{t}\right)\right]=0 .
\end{aligned}
$$

Integrating the local momentum conservation law in Eq. (17) over the spatial domain, the global momentum conservation law can be obtained as

$$
\frac{1}{4} \frac{\mathrm{d}}{\mathrm{d} t} \int\left(-u_{x} \varphi+u_{x} v+u \varphi_{x}-u v_{x}\right) \mathrm{d} x+\left.\left[S(\mathbf{z})+\frac{1}{4}\left(\varphi u_{t}-v u_{t}-u \varphi_{t}+u v_{t}\right)\right]\right|_{l_{1}} ^{l_{2}}=0
$$

where $l_{1}$ and $l_{2}$ denote the upper and lower limits of the integral. Using the canonical momenta relations in Eq. (7), after two integrations by parts, Eq. (18) becomes

$$
\frac{1}{2} \frac{\mathrm{d}}{\mathrm{d} t} \int\left(u^{2}+u_{x}^{2}\right) \mathrm{d} x+\left.[D]\right|_{l_{1}} ^{l_{2}}=0
$$

where $D=S(\mathbf{z})+\frac{1}{4}\left(\varphi u_{t}-v u_{t}-u \varphi_{t}+u v_{t}\right)-\frac{1}{4}\left(u \varphi+u u_{x}\right)_{t}$.

Under the periodic boundary conditions, the value of $\left.[D]\right|_{l_{1}} ^{l_{2}}$ is zero. Thus, the global conserved quantity $H_{1}$ is just the global conserved momentum quantity associated with 
the Camassa-Holm equation. Similarly, integrating the local energy conservation law in Eq. (17) over the spatial domain, the global energy conservation law can be obtained, which is just the global conserved quantity $\mathrm{H}_{2}$ for the Camassa-Holm equation.

In Section 4.1 we find that the values of $u$ approach to zero when $|x|>15$, and thus the three conserved quantities (16) can be approximated as

$$
\left\{\begin{array}{l}
H_{0}=\int_{-15}^{15} u \mathrm{~d} x \\
H_{1}=\frac{1}{2} \int_{-15}^{15}\left(u^{2}+u_{x}^{2}\right) \mathrm{d} x \\
H_{2}=\frac{1}{2} \int_{-15}^{15}\left(u^{3}+u u_{x}^{2}\right) \mathrm{d} x
\end{array}\right.
$$

Calculating Eq. (20), the values of the three conserved quantities can be obtained:

$$
H_{0}=2-2 e^{-15}, \quad H_{1}=1-e^{-30}, \quad H_{2}=\frac{2}{3}-\frac{2}{3} e^{-45} .
$$

It is well known that the multisymplectic method can preserve the inherent local geometric properties of infinite-dimensional Hamiltonian systems, so we would like to know whether the quantities mentioned above are conserved. The relative errors in $H_{0}, H_{1}$, and $\mathrm{H}_{2}$ of the multisymplectic Euler box scheme (10) about the three conserved quantities (20) on each grid point are recorded, and the integration of which with respect to $x$ is obtained to show the preserving status of the conserved quantities in the Camassa-Holm equation.

Similarly, we take the time step length $\Delta x=0.005$ and space step length $\Delta t=0.005$ in the domain $D:(t, x) \in[0,2] \times[-15,15]$. Considering the initial condition (15), the numerical values of $u$ can be obtained firstly. The discrete conserved quantities (20) of the Camassa-Holm equation can be simplified as follows:

$$
\left\{\begin{array}{l}
H_{0}=\int_{-15}^{15} u \mathrm{~d} x \approx \Delta x \sum_{n=1}^{N} u^{n, i} \\
H_{1}=\frac{1}{2} \int_{-15}^{15}\left(u^{2}+u_{x}^{2}\right) \mathrm{d} x \approx \frac{\Delta x}{2} \sum_{n=1}^{N}\left[\left(u^{n, i}\right)^{2}+\left(\frac{u^{n+1, i}-u^{n-1, i}}{2 \Delta x}\right)^{2}\right] \\
H_{2}=\frac{1}{2} \int_{-15}^{15}\left(u^{3}+u u_{x}^{2}\right) \mathrm{d} x \approx \frac{\Delta x}{2} \sum_{n=1}^{N}\left[\left(u^{n, i}\right)^{3}+u^{n, i} \cdot\left(\frac{u^{n+1, i}-u^{n-1, i}}{2 \Delta x}\right)^{2}\right]
\end{array}\right.
$$

Then, according to Eq. (22), the discretization form of the three conserved quantities can be calculated. The relative errors are defined by

$$
\operatorname{Re}_{i}=\frac{\left|H_{i}-H_{i}(t)\right|}{H_{i}(t)}, \quad i=0,1,2
$$

where $H_{i}(t)$ are the numerical solutions of the conserved quantities $H_{i}$ at $t \in[0,2]$.

The numerical solution in a larger spatial domain of the traveling peaked wave is given in Figure 3, which is similar to the results given in Section 4.1, that is, the wave shape is smooth enough in the process of the propagation. The errors between numerical solution and exact solution are in the same order of magnitude shown in Figure 2; thus, these results are not presented here.

The relative errors of the conserved quantities (20) according to the definition (23) are presented in Figure 4. From Figure 4 we can find that the relative values of the errors of the conserved quantities (20) are less than $5 \times 10^{-3}$, which implies that the three global conserved quantities (16) are preserved in the Camassa-Holm equation and proves that the numerical results contain some inherent geometric properties of the Camassa-Holm 

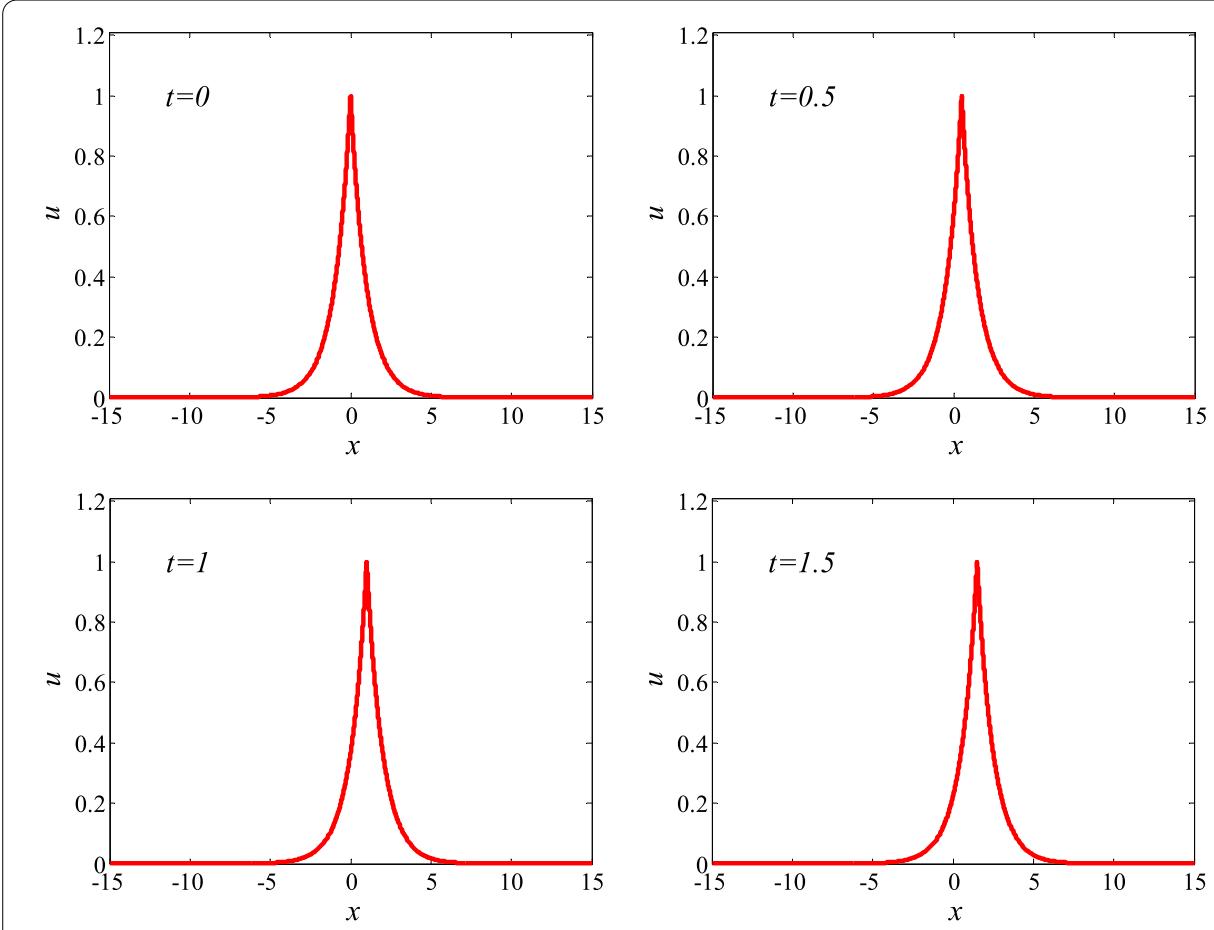

Figure 3 Waveforms of the peakon solution.

Figure 4 Relative errors of the conserved quantities.
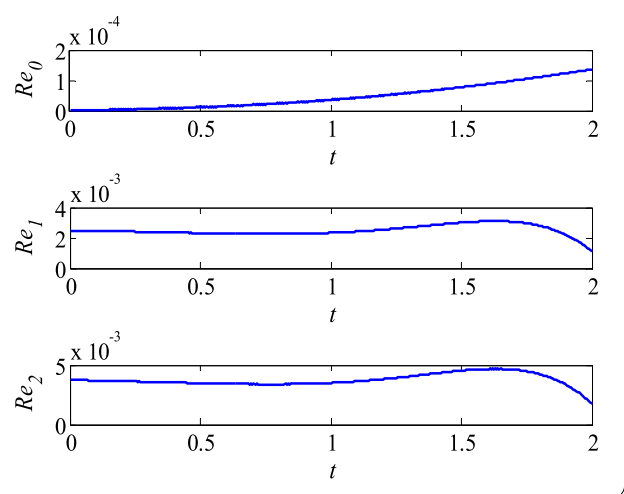

equation. The small errors of the conserved quantities (20) illustrate that the multisymplectic Euler box scheme (10) has good numerical behavior and can be used to simulate the traveling peaked wave solution of the Camassa-Holm equation.

\section{Conclusions}

In this paper, the multisymplectic method, a numerical method that can preserve the local geometric properties of the Hamiltonian systems, is used to simulate the peakon of the Camassa-Holm equation.

By introducing the suitable canonical momenta the Camassa-Holm equation is first transformed into the multisymplectic structure. Then, based on the Euler box method, a multisymplectic discrete scheme is constructed. The numerical results of the peaked 
wave of the Camassa-Holm are presented. From the numerical results we can conclude that:

1. The multisymplectic Euler box method can well simulate the traveling peaked wave of the Camassa-Holm equation with small errors. The numerical simulation results also verified that the soliton has a discontinuity in the first derivative at its peak. These results illustrate the structure-preserving properties of the multisymplectic integration.

2. The numerical results on the three conserved quantities of the peakon imply that the multisymplectic method can preserve the inherent geometric properties of the Camassa-Holm equation.

\section{Competing interests}

The authors declare that they have no competing interests.

\section{Authors' contributions}

All authors completed the paper together. All authors read and approved the final manuscript.

\section{Acknowledgements}

The research is supported by the National Natural Science Foundation of China (11372252 and 11372253), the National Basic Research Program of China 973 (2011CB610300), the PhD Programs Foundation of Ministry of Education of China (20126102110023), and the Fundamental Research Funds for the Central Universities (3102014JCQ01035).

Received: 10 July 2015 Accepted: 19 November 2015 Published online: 07 January 2016

\section{References}

1. Holm, DD, Staley, MF: Nonlinear balance and exchange of stability in dynamics of solitons, peakons, ramps/cliffs and leftons in a $1+1$ nonlinear evolutionary PDE. Phys. Lett. A 308, 437-444 (2003)

2. Camassa, R, Holm, DD: An integrable shallow-water equation with peaked solitons. Phys. Rev. Lett. 71, 1661-1664 (1993)

3. Constantin, A: On the scattering problem for the Camassa-Holm equation. Proc. R. Soc. Lond. A 457, $953-970$ (2001)

4. Fisher, M, Schiff, J: The Camassa Holm equation: conserved quantities and the initial value problem. Phys. Lett. A 259, 371-376 (1999)

5. Degasperis, A, Holm, DD, Hone, ANW: A new integrable equation with peakon solutions. Theor. Math. Phys. 133, 1463-1474 (2002)

6. Lundmark, HJ: Formation and dynamics of shock waves in the Degasperis-Procesi equation. J. Nonlinear Sci. 17 169-198 (2007)

7. Coclite, GM, Karlsen, $\mathrm{KH}$, Risebro, NH: Numerical schemes for computing discontinuous solutions of the Degasperis-Procesi equation. IMA J. Numer. Anal. 28, 80-105 (2008)

8. Coclite, GM, Gargano, F, Sciacca, V: Analytic solutions and singularity formation for the peakon $b$-family equations. Acta Appl. Math. 122, 419-434 (2012)

9. Coclite, GM, Karlsen, $\mathrm{KH}$, Risebro, $\mathrm{NH}$ : A convergent finite difference scheme for the Camassa-Holm equation with general $H^{1}$ initial data. SIAM J. Numer. Anal. 46, 1554-1579 (2008)

10. Coclite, GM, Karlsen, $\mathrm{KH}$, Risebro, NH: An explicit finite difference scheme for the Camassa-Holm equation. Adv. Differ. Equ. 13,681-732 (2008)

11. Xu, Y, Shu, CW: A local discontinuous Galerkin method for the Camassa-Holm equation. SIAM J. Numer. Anal. 46, 1998-2021 (2008)

12. Matsuo, T: A Hamiltonian-conserving Galerkin scheme for the Camassa-Holm equation. J. Comput. Appl. Math. 234, 1258-1266 (2010)

13. Kalisch, H, Lenells, J: Numerical study of traveling-wave solutions for the Camassa-Holm equation. Chaos Solitons Fractals 25, 287-298 (2005)

14. Kalisch, H, Raynaud, X: Convergence of a spectral projection of the Camassa-Holm equation. Numer. Methods Partial Differ. Equ. 22, 1197-1215 (2006)

15. Cohen, D, Owren, B, Raynaud, X: Multi-symplectic integration of the Camassa-Holm equation. J. Comput. Phys. 227, 5492-5512 (2008)

16. Zhu, HJ, Song, SH, Tang, YF: Multi-symplectic wavelet collocation method for the nonlinear Schrödinger equation and the Camassa-Holm equation. Comput. Phys. Commun. 182, 616-627 (2011)

17. Bridges, TJ: Multi-symplectic structures and wave propagation. Math. Proc. Camb. Philos. Soc. 121, 147-190 (1997)

18. Reich, S: Multi-symplectic Runge-Kutta collocation methods for Hamiltonian wave equations. J. Comput. Phys. 157, 473-499 (2000)

19. Bridges, TJ, Reich, S: Multi-symplectic integrators: numerical schemes for Hamiltonian PDEs that conserve symplecticity. Phys. Lett. A 284, 184-193 (2001)

20. Wang, YS, Wang, B: High-order multi-symplectic schemes for the nonlinear Klein-Gordon equation. Appl. Math. Comput. 166, 608-632 (2005)

21. Li, HC, Sun, JQ, Qin, MZ: New explicit multi-symplectic scheme for nonlinear wave equation. Appl. Math. Mech. 35 , $369-380(2014)$ 
22. Hong, JL, Jiang, SS, Li, C: Explicit multi-symplectic methods for Klein-Gordon-Schrödinger equations. J. Comput. Phys. 228, 3517-3532 (2009)

23. Hong, JL, Kong, LH: Novel multi-symplectic integrators for nonlinear fourth-order Schrödinger equation with trapped term. Commun. Comput. Phys. 7, 613-630 (2010)

24. Qian, X, Chen, YM, Gao, E, Song, SH: Multi-symplectic wavelet splitting method for the strongly coupled Schrödinger system. Chin. Phys. B 21, 120202 (2012)

25. Lv, ZQ, Wang, YS, Song, YZ: A new multi-symplectic integration method for the nonlinear Schrödinger equation. Chin. Phys. Lett. 30, 030201 (2013)

26. Zhao, PF, Qin, MZ: Multisymplectic geometry and multisymplectic Preissmann scheme for the KdV equation. J. Phys. A, Math. Gen. 33, 3613-3626 (2000)

27. Wang, YS, Wang, B, Chen, X: Multisymplectic Euler box scheme for the KdV equation. Chin. Phys. Lett. 24, 312-314 (2007)

28. Hu, WP, Deng, ZC: Multi-symplectic method for generalized fifth-order KdV equation. Chin. Phys. B 17, 3923-3929 (2008)

29. Zeng, WP, Huang, LY, Qin, MZ: The multi symplectic algorithm for 'good' Boussinesq equation. Appl. Math. Mech. 23 835-841 (2002)

30. Hu, WP, Deng, ZC: Multi-symplectic method for generalized Boussinesq equation. Appl. Math. Mech. 29, 927-932 (2008)

31. Kong, LH, Hong, JL, Zhang, JJ: Splitting multisymplectic integrators for Maxwell's equations. J. Comput. Phys. 229 4259-4278 (2010)

32. Cai, WJ, Wang, YS, Song, YZ: Numerical dispersion analysis of a multi-symplectic scheme for the three dimensional Maxwell's equations. J. Comput. Phys. 234, 330-352 (2013)

33. Hong, JL, Ji, LH, Zhang, LY: A stochastic multi-symplectic scheme for stochastic Maxwell equations with additive noise. J. Comput. Phys. 268, 255-268 (2014)

34. Hu, WP, Deng, ZC, Zhang, Y: Multi-symplectic method for peakon-antipeakon collision of quasi-Degasperis-Procesi equation. Comput. Phys. Commun. 185, 2020-2028 (2014)

35. Cai, WJ, Wang, YS: A new explicit multisymplectic integrator for the Kawahara-type equation. Chin. Phys. B 23, 030204 (2014)

36. Moore, B, Reich, S: Backward error analysis for multi-symplectic integration methods. Numer. Math. 95, 625-652 (2003)

37. Liu, Y, Yin, ZY: Global existence and blow-up phenomena for the Degasperis-Procesi equation. Commun. Math. Phys. $267,801-820(2006)$

\section{Submit your manuscript to a SpringerOpen ${ }^{\mathcal{O}}$ journal and benefit from:}

- Convenient online submission

Rigorous peer review

- Immediate publication on acceptance

- Open access: articles freely available online

- High visibility within the field

- Retaining the copyright to your article 